\title{
Laser cooling of trapped atoms to the ground state: A dark state in position space
}

\author{
G. Morigi, J. I. Cirac, K. Ellinger, and P. Zoller \\ Institut für Theoretische Physik, Universität Innsbruck, A-6020 Innsbruck, Austria
}

(Received 14 October 1997)

\begin{abstract}
We propose a scheme that allows us to laser cool trapped atoms to the ground state of a one-dimensional confining potential. The scheme is based on the creation of a dark state by designing the laser profile, so that the hottest atoms are coherently pumped to another internal level, and then repumped back. The scheme works beyond the Lamb-Dicke limit. We present results of a full quantum treatment for a one-dimensional model. [S1050-2947(98)10104-X]
\end{abstract}

PACS number(s): $32.80 . P \mathrm{j}, 42.50 . \mathrm{Vk}$

\section{INTRODUCTION}

The ultimate goal of laser cooling neutral atoms stored in a trapping potential is the cooling to the vibrational ground state. This is of interest in the context of observing effects related to quantum-statistical properties of atoms [1], and is the object of present experimental investigations [2,3]. The hope is that these efforts might ultimately open a second route to Bose-Einstein condensation of dilute gases [4,5].

Laser cooling to the ground state of a trap has been achieved experimentally for single-trapped ions, using sideband cooling techniques [6] in the Lamb-Dicke limit [7,8]. Sideband cooling is based on the selective laser excitation of the low-frequency sideband in a harmonic trap, leading to optical pumping into the vibrational ground state. The requirements are that the motional sidebands are spectroscopically resolved (which is achieved in the strong confinement limit), and that the spatial dimension of the ground state $a_{0}$ is much smaller than the wavelength of the cooling laser $\lambda$ (in order to avoid the heating produced by spontaneous emission). This latter condition is represented by the relation $\eta \ll 1$, where $\eta$ is the Lamb-Dicke parameter $\eta=2 \pi a_{0} / \lambda$. In one of our recent publications [9], we discussed extensions of sideband cooling beyond the Lamb-Dicke regime. Over the last few years, other laser cooling techniques have been developed, which achieve ground-state cooling in the trapping potential, and which were originally developed for free atoms $[2,3]$. In particular, for free atoms they have allowed one to achieve temperatures below the recoil limit $E<E_{R}$, corresponding to an atomic de Broglie wavelength larger than the wavelength of the light $\lambda$. This has been obtained experimentally, by optical pumping into a velocity-selective dark state [10], or by cooling with a sequence of shaped Raman pulses, where the frequency spectrum of the light is tailored so that atoms with near zero velocity are no longer excited [11]. These subrecoil cooling techniques, as well as sideband cooling, are all versions of "dark state cooling." The fundamental idea is the decoupling of a quantum state from radiation, and the accumulation of atoms in this state by spontaneous emission. The dark state condition can be created with various mechanisms; in sideband cooling, the trap ground state is dark because it is off-resonant from any other state, since the laser is tuned to the red motional sideband. The free-particle subrecoil cooling schemes, instead, use dark states in momentum space.
In the present paper we will discuss a cooling scheme which is based on the creation of a dark state in position space with the help of an appropriate spatial profile of the cooling laser, so that this state (typically the ground state of the trap) is not excited or only weakly excited by the laser [12]. This condition can be realized, for example, using the laser in a doughnut mode [13], with the axis aligned with one of the trap axes. In particular, this cooling mechanism works for Lamb-Dicke parameters $\eta \geqslant 1$, and outside of the strong confinement regime (i.e., when the sidebands are not spectroscopically resolved). We will show that the cooling scheme is quite efficient, allowing us to cool a significant fraction of atoms to the ground state of a trapping potential. The model we will consider is one dimensional, but the scheme is readily extended to two- and three-dimensional situations.

The paper is organized as follows. In Sec. II A, we describe the cooling scheme qualitatively. In Sec. II B we develop the master equation describing the system dynamics, and in Sec. II C we discuss the existence of a stationary solution. In Sec. III we present the numerical results, obtained by taking a laser intensity profile corresponding to a doughnut mode with (i) atoms trapped by an harmonic oscillator, and (ii) atoms trapped by another confining potential. In this last case, the efficiency of the cooling mechanism is improved.

\section{MODEL}

\section{A. Laser-cooling scheme}

We consider laser cooling in a three-level $\Lambda$ system, as illustrated in Fig. 1. We denote by $|g\rangle$ and $|e\rangle$ atomic ground (or metastable) states which are connected to the excited state $|r\rangle$ by dipole transitions. The atoms are confined in a one-dimensional harmonic potential with oscillation frequency $\nu$. The quantized trap levels will be denoted by $|n\rangle$.

The laser-cooling scheme consists of the repetition of a sequence of three pulses. In a first step, atoms in $|g\rangle$ are transferred to the state $|e\rangle$ by an off-resonant Raman pulse [Fig. 1(a)]. The purpose of this pulse is to excite atoms which are not in the ground state of the trapping potential. This is achieved by assuming a spatial distribution of the laser, which has essentially zero intensity in the region where the ground-state wave function $|g, 0\rangle$ takes on appreciable values. On the other hand, the spatial distribution of atoms in 

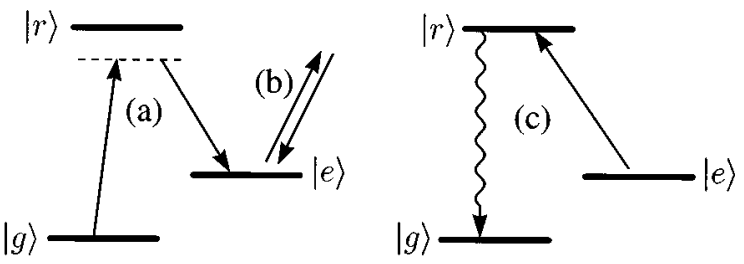

FIG. 1. Internal configuration of the atom, and scheme of the sequence of pulses. Internal configuration: $|g\rangle,|e\rangle$ stable or metastable states, $|r\rangle$ excited state, with $|g\rangle \rightarrow|r\rangle,|e\rangle \rightarrow|r\rangle$ dipole transitions. Scheme of the sequence: (a) coherent Raman pulse, which corresponds to a doughnut mode on $|g\rangle \rightarrow|r\rangle$; (b) cooling to the recoil on the level $|e\rangle$, obtained coupling $|e\rangle$ to a fourth internal level; (c) optical pumping into $|g\rangle$, obtained tuning a laser on resonance on $|e\rangle \rightarrow|r\rangle$. We assume that the branching ratio between $|r\rangle \rightarrow|g\rangle$ and $|r\rangle \rightarrow|e\rangle$ is very large, so that the decay along $|r\rangle$ $\rightarrow|e\rangle$ can be safely neglected.

excited trap states overlaps with the laser light, so that these atoms will be transferred to the state $|e\rangle$. The second step consists of laser cooling atoms in state $|e\rangle$, thermalizing the atomic center of the mass distribution to a few recoil energies $E_{R}$ [Fig. 1(b)]. This is achieved by coupling $|e\rangle$ to other internal atomic levels employing one of the standard lasercooling schemes (e.g., polarization gradient cooling) [14]. This is used to provide a mechanism for confinement of atoms in a finite range of trapping levels. In a third step, atoms are optically pumped into $|g\rangle$ using a laser tuned on resonance to the transition $|e\rangle \rightarrow|r\rangle$ followed by spontaneous transition to $|g\rangle$ [Fig. 1(c)]. We assume that the branching ratio between the decay channels $|r\rangle \rightarrow|g\rangle$ and $|r\rangle \rightarrow|e\rangle$ is large, so that we can safely neglect the latter decay channel. Atoms decaying from $|r\rangle$ to the ground state $|g\rangle$ will be distributed over a range of trap levels of the order of a recoil energy, including the ground state $|g, 0\rangle$. Repetition of this cooling cycle will accumulate atoms in the spatial dark state $|g, 0\rangle$.

\section{B. Master equation}

Here we give the master equation describing the evolution of the system during the sequence of pulses. Let $\rho$ be the density matrix describing the system of atoms interacting with the laser field. The master equation can be written in the form [15]

$$
\frac{d}{d t} \rho(t)=L \rho(t)
$$

where $L$ is a linear superoperator whose form depends on the particular cooling step. The formal solution is $\rho(t)=e^{L\left(t-t_{0}\right)} \rho\left(t_{0}\right)$. The scheme consists of three steps, and the density matrix at the end of a sequence of pulses will be

$$
\rho\left(t_{3}\right)=e^{L_{3}\left(t_{3}-t_{2}\right)} e^{L_{2}\left(t_{2}-t_{1}\right)} e^{L_{1}\left(t_{1}-t_{0}\right)} \rho\left(t_{0}\right),
$$

where $L_{i}$ is the linear superoperator corresponding to the $i$ th pulse of the sequence, and $\rho\left(t_{0}\right)$ is the density matrix at the beginning of the sequence of pulses. We now derive the explicit form of the evolution operators.

First step: We assume that the evolution is coherent, and that the lasers are in Raman resonance. These two assump- tions are not strictly necessary, but they make the analytical derivation of the master equation simpler. We eliminate the excited state $|r\rangle$ in perturbation theory, and reduce the dynamics to an effective two-level system $|g\rangle,|e\rangle$. Working in the frame rotating at the laser frequency, we write

$$
\rho\left(t_{1}\right)=e^{-i H\left(t_{1}-t_{0}\right)} \rho\left(t_{0}\right) e^{i H\left(t_{1}-t_{0}\right)},
$$

where $H$ is the Hamiltonian describing the evolution during the pulse,

$$
H=H_{0}+H_{\mathrm{I}},
$$

with

$$
H_{0}=\hbar \nu \hat{a}^{\dagger} \hat{a}
$$

and

$$
H_{\mathrm{I}}=\frac{\hbar \Omega(\hat{x})}{2}\left(\hat{\sigma}^{\dagger} e^{i k \hat{x}}+\hat{\sigma} e^{-i k \hat{x}}\right)
$$

Here $\hat{a}$ and $\hat{a}^{\dagger}$ are creation and annihilation operators for the harmonic oscillator, $\nu$ is the trap frequency, $\hat{\sigma}^{\dagger}=|e\rangle\langle g|$ and $\hat{\sigma}=|g\rangle\langle e|$ are the dipole raising and lowering operators, respectively, $\Omega(\hat{x})$ is the Rabi frequency, and $k$ the wave vector of the two-photon transition along the (cooling) $x$ axis. Denoting by $\Delta t=t_{1}-t_{0}$ the time duration of this first pulse, we will assume that $\nu \Delta t \ll 1$, so that we can safely neglect $H_{0}$ in Eq. (3):

$$
\rho\left(t_{1}\right) \approx e^{-i H_{\mathrm{I}} \Delta t} \rho\left(t_{0}\right) e^{i H_{\mathrm{I}} \Delta t} .
$$

Second step: Laser cooling of atoms in state $|e\rangle$ via an auxiliar level [see Fig. 1(b)] destroys the coherences between $|e\rangle$ and $|g\rangle$, while atoms left in $|g\rangle$ evolve according to Eq. (5). We do not specify the cooling mechanism, assuming only that at the end of the pulse the atoms in $|e\rangle$ are thermalized, and described by the density matrix

$$
|e\rangle\left\langle e\left|\otimes \rho^{\mathrm{th}}=\right| e\right\rangle\langle e| \otimes \frac{\sum_{n} e^{-n / N}|n\rangle\langle n|}{\sum_{n} e^{-n / N}},
$$

where $N$ is related to the average number of vibrational quanta after this laser-cooling process. Using the properties of $\hat{\sigma}^{\dagger}$ and $\hat{\sigma}$ in Eq. (6), we have

$$
\begin{aligned}
\rho\left(t_{2}\right)= & e^{-i H_{0} T_{\mathrm{sep}}}|g\rangle\langle g| \cos [\Omega(\hat{x}) \Delta t / 2] \rho\left(t_{0}\right) \\
& \times \cos [\Omega(\hat{x}) \Delta t / 2]|g\rangle\left\langle g\left|e^{i H_{0} T_{\mathrm{sep}}}+\zeta\right| e\right\rangle \rho^{\mathrm{th}}\langle e|,
\end{aligned}
$$

where $T_{\text {sep }}=t_{2}-t_{1}$ is the duration of this step, and $\zeta$ is the probability of occupation of $|e\rangle$, i.e.,

$$
\begin{aligned}
\zeta= & \operatorname{trace}\left\{|e\rangle\langle e| \rho\left(t_{1}\right)\right\} \\
= & 1-\operatorname{trace}\left\{|g\rangle\langle g| \cos [\Omega(\hat{x}) \Delta t / 2] \rho\left(t_{0}\right)\right. \\
& \times \cos [\Omega(\hat{x}) \Delta t / 2]\} .
\end{aligned}
$$


Third step: the thermalized atoms in $|e\rangle$ are optically pumped into $|g\rangle$. The linear superoperator describing the master equation is

$$
\begin{aligned}
L_{3} \rho= & -i\left[H_{0}, \rho\right]-\frac{\Gamma}{2}\left(\rho \hat{\sigma}^{\dagger} \hat{\sigma}+\hat{\sigma}^{\dagger} \hat{\sigma} \rho\right) \\
& +\Gamma \int_{-1}^{1} d u N(u) \hat{\sigma} e^{i k_{p}(1+u) \hat{x}} \rho^{\text {th }} e^{-i k_{p}(1+u) \hat{x}} \hat{\sigma}^{\dagger},
\end{aligned}
$$

with $\Gamma$ the optical pumping rate, $N(u)$ the angular distribution of the emitted photons, and $k_{p}$ the wave vector of the pumping laser that propagates along $x, k_{p}=2 \pi / \lambda=\eta / a_{0}$. Assuming $\nu^{-1} \gg t_{3}-t_{2}$, we can neglect the free evolution during this pulse. Furthermore, for $t_{3}-t_{2} \gg \Gamma^{-1}$ all the atoms in $|e\rangle$ are pumped in $|g\rangle$ during the pulse. Taking that into account, we find

$$
\begin{aligned}
& \rho\left(t_{3}\right)=e^{-i H_{0} T_{\text {sep }}}|g\rangle\langle g| \cos [\Omega(\hat{x}) \Delta t / 2] \rho\left(t_{0}\right) \cos [\Omega(\hat{x}) \\
& \times \Delta t / 2]|g\rangle\left\langle g\left|e^{i H_{0} T_{\mathrm{sep}}+\zeta} \int_{-1}^{1} d u N(u) e^{i k_{p}(1+u) \hat{x}}\right| g\right\rangle \\
& \times \rho^{\mathrm{th}}\langle g| e^{-i k_{p}(1+u) \hat{x}} \text {. }
\end{aligned}
$$

We can now project onto $|g\rangle$, and define $\rho_{g}=\langle g|\rho| g\rangle$. Denoting by $F=\int_{-1}^{1} d u N(u) e^{i k_{p}(1+u) \hat{x}} \rho^{\text {th }} e^{-i k_{p}(1+u) \hat{x}}$ the matrix describing the feeding contribution, we finally have

$$
\begin{aligned}
\rho_{g}\left(t_{3}\right)= & e^{-i H_{0} T_{\text {sep }}} \cos [\Omega(\hat{x}) \Delta t / 2] \rho_{g}\left(t_{0}\right) \\
& \times \cos [\Omega(\hat{x}) \Delta t / 2] e^{i H_{0} T_{\mathrm{sep}}+\zeta F} .
\end{aligned}
$$

This gives a mapping between the density operator at the beginning and end of a given sequence of three pulses. From the above expressions, we can deduce that for large traps (i.e., for large values of the Lamb-Dicke parameter) the cooling will slow down and become less efficient. The reason for this is twofold: On the one hand, the kick provided by the optical pumping process will distribute the atoms among a wider range of states. On the other hand, since we have assumed that the thermal distribution $\rho^{\text {th }}$ has a mean energy of the order of the recoil, the mean occupation number will be larger $\left(\langle n\rangle \approx E_{R} / \hbar \nu=\eta^{2}\right)$.

\section{Stationary state}

Ideally, we would like to use the cooling mechanism to cool all the atoms to the ground state (or any other pure state). Therefore, we have to analyze the shape of $\Omega(x)$ which gives rise to the optimal cooling. In this subsection we qualitatively analyze the conditions for the spatial profile of the laser $\Omega(x)$ to provide an efficient cooling.

We then look for stationary solutions to the mapping (13) which corresponds to pure states. A necessary condition for that is $\zeta=0$. Then, we substitute $\zeta=0$ in Eq. (13) and impose the stationary regime, i.e., $\rho_{g}\left(t_{3}\right)=\rho_{g}\left(t_{0}\right)=\rho_{g}$, with $\rho_{g}$ $=|f\rangle\langle f|$. We obtain that these conditions are satisfied if the following equation allows for a solution

$$
\left\langle x\left|e^{i H_{0} T_{\mathrm{sep}}}\right| f\right\rangle e^{i \phi}=\cos [\Omega(x) \Delta t / 2]\langle x \mid f\rangle,
$$

with $\phi$ arbitrary phase. Let us denote by $R$ the region where $\langle x \mid f\rangle$ is different from zero. It can be easily shown that Eq. (14) implies that in the region $R$ the relation $|\cos [\Omega(x) \Delta t / 2]|=1$ must be fulfilled. Physically, this means that if we want to have a dark state $|f\rangle$ its wave function must be completely distributed in the spatial region $R$ where there is no laser excitation (that is, where the atoms perform complete Rabi oscillations). On the other hand, we want to have only one dark state, and therefore other states must have a finite excitation probability. This means that they must be nonzero outside $R$. Therefore, for achieving cooling to the ground state, one should design the intensity profile of the laser in such a way that, only in the region where the ground state is localized, the laser accomplishes no excitation. This condition can be only approximately fulfilled in a harmonic trap, where the ratio among the spatial dimensions of the ground state and the first excited state is $1 / \sqrt{2}$. In this case, we expect to not be able to cool all the atoms into the ground state. Cooling into the ground state will be possible in the case of atoms trapped by a confining potential, which better localizes the ground state with respect to the first excited state.

The qualitative argument given above does not depend on the separation time $T_{\text {sep }}$ in the second pulse. In reality, the selection of this time can play an important role, especially in the case of a harmonic potential. To see that, let us consider the particular case $\nu T_{\text {sep }}=2 l \pi$, with $l$ an integer. Then, according to Eq. (13), the positions $x$ in the region $R$ in which $|\cos [\Omega(x) \Delta t / 2]| \neq 1$ will be emptied, whereas the atoms will be accumulated in the regions where $|\cos [\Omega(x) \Delta t / 2]|=1$. In fact, from Eq. (14), we see that the state $|f\rangle$ is a stable solution if the conditions $\cos [\Omega(x) \Delta t / 2]|f\rangle=\exp \left(i \phi_{1}\right)|f\rangle$ and if $\exp \left(-i H_{0} T_{\text {sep }}\right)|f\rangle$ $=\exp \left(i \phi_{2}\right)|f\rangle$ are fulfilled, with $\phi_{1}+\phi_{2}=\phi$. For $\nu T_{\text {sep }}=2 l \pi$ this means that each wave packet completely distributed inside $R$ at $t_{0}$ is solution (i.e., a dark state), since in $T_{\text {sep }}$ it undergoes a full oscillation and therefore recovers the original form. In that case, laser cooling will not be possible since there will exist many wavepackets that will remain dark. In the case $\nu T_{\text {sep }}=(2 l+1) \pi$, one can consider two subspaces $S_{1}=\{|0\rangle,|2\rangle,|4\rangle, \ldots\}$ and $S_{2}=\{|1\rangle,|3\rangle,|5\rangle, \ldots\}$, in which the evolution during the second pulse is periodic. That is, if we can form a wave packet as a linear combination of the states of $S_{1}$ or of $S_{2}$ which is spatially distributed in $R$ at $t_{0}$, this wave packet will be a dark state. Hence laser cooling will not be possible either. The argument can now be repeated for times fulfilling $\nu T_{\text {sep }}=n \pi / m$, and subspaces can be constructed so that wavepackets belonging to them will be dark states, and therefore laser cooling will not be possible. We can overcome this problem very easily by choosing random separation times $T_{\text {sep }}$, so that the only wave packet that is a dark state is precisely the ground state.

\section{RESULTS OF CALCULATIONS}

In the following, we show results obtained assuming a doughnut mode as the spatial distribution of the Raman pulse. The reason for this choice is that it provides a flat profile in a finite region which, as we have shown above, is required to obtain a suitable cooling. In particular, we discuss the parameter regime of the laser for which the cooling 


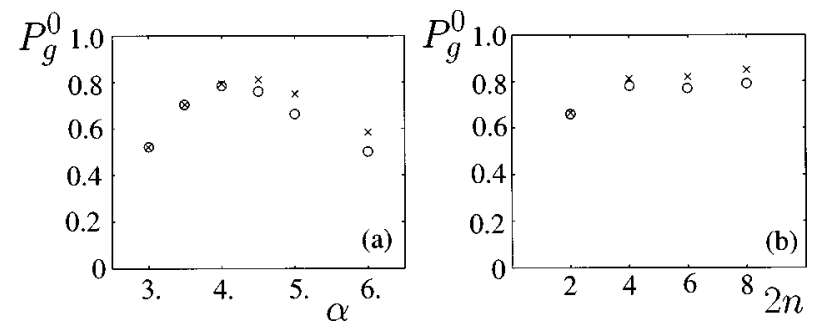

FIG. 2. Plots of the efficiency after 1500 (curve with $\bigcirc$ ) and after 2500 (curve with $\times$ ) sequences of pulses, as a function of (a) the dimensionless width $\alpha$, for $2 n=4$; and (b) the exponents $2 n=2,4,6,8$ with optimized widths, respectively, where $\alpha=30,4.2$, 3 , and 2.2. In all cases $\max [\Omega(x) \Delta t / 2]=0.6 \pi$. $\nu T_{\text {sep }}$ is random, taken from a flat distribution that varies in the interval $[0.1,1.1]$.

efficiency is optimal. Finally, we explore the role of $T_{\text {sep }}$ in the cooling dynamics. We first analyze the case of a harmonic trapping potential and then another potential which is best suited for laser cooling using the present scheme.

As the spatial distribution for the Raman pulse laser, we take the following class of functions:

$$
f(x)=\left(\frac{x}{a_{0}}\right)^{2 n} \exp \left[-\frac{1}{2}\left(\frac{x / a_{0}}{\alpha}\right)^{2}\right] .
$$

Here $\alpha$ determines the spatial width of the pulse in units of the harmonic-oscillator ground-state width $a_{0}$, and the exponent $n$ is an integer number, which denotes the order of the doughnut mode and thus determines the flatness around $x=0$. In the following, we explore the dependence of the cooling efficiency on the parameters $n$ and $\alpha$, for atoms whose center of mass is trapped by a harmonic potential with frequency $\nu$. We take the Rabi frequency $\Omega(\hat{x}) \propto f(\hat{x})$. Furthermore, the projection along the cooling axis $(x)$ of the two-photon wave vector appearing in Eq. (6) is $k=2 \pi / \lambda$, where $\lambda$ is the wavelength of the transition $|r\rangle \rightarrow|e\rangle$, since the doughnut mode photons propagate in a direction orthogonal to the $x$ axis.

In Figs. 2(a) and 2(b), we show the population $P_{g}^{0}=\left\langle g, 0\left|\rho_{g}\right| g, 0\right\rangle$ after 1500 (curve with $\bigcirc$ ) and 2500 (curve with $\times$ ) sequences of pulses as a function of the width $\alpha$ and of the exponent $2 n$, respectively. We have obtained these results using Eq. (13), with a Lamb-Dicke parameter $\eta=2 \pi a_{0} / \lambda=5$, and with a thermalized distribution $\rho^{\text {th }}$ in Eq. (8) with $N=\eta^{2}=25$. From Fig. 2(a) the existence of an optimal width $\alpha$ for a given exponent (in the present case, $2 n=4$ ) is evident. The optimal $\alpha$ depends on $n$, since it must fulfill the requirements of Eq. (14). In Fig. 2(b) we plot $P_{g}^{0}$ as a function of $2 n$, where the values of the width have been optimized. We see that for $n \geqslant 4$, around $80 \%$ of the atoms are cooled to the ground state. Figure 3(a) shows the population $P_{g}^{n}=\left\langle n\left|\rho_{g}\right| n\right\rangle$ as a function of the vibrational number $n$ after 2500 sequences of pulses for $n=4$ and $\alpha=4$, and Fig. 3(b) shows $P_{g}^{0}$ as a function of the number of pulses. In the inset of this figure the final spatial distribution is shown (solid line), while the spatial distribution $\cos [\Omega(x) \Delta t / 2]$ is plotted as a dashed line for comparison. In all the cases, the final density matrix is not a pure state but a mixture, diagonal in the number states basis. According to the discussion in Sec. II, we have chosen random separation times. We have
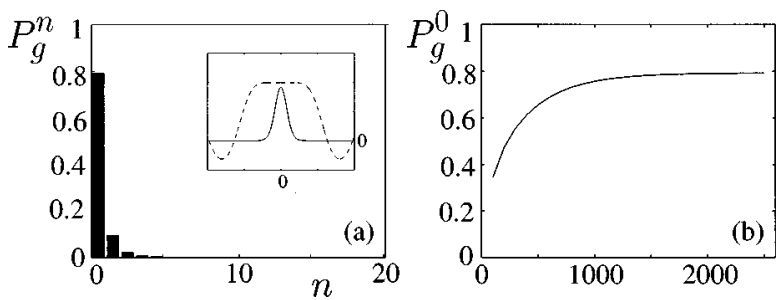

FIG. 3. (a) Population as a function of the vibrational number state after 2500 sequences of pulses, for a harmonic oscillator with $\eta=5$ and Raman laser corresponding to a doughnut mode with $2 n=4, \alpha=4$, and $\max [\Omega(x) \Delta t / 2]=0.6 \pi$. Inlay: spatial distribution of the sample after 2500 pulses (solid line) and $\cos [\Omega(x) \Delta t / 2]$ (dashed line) plotted for comparison. (b) Ground state of the trap population as a function of the number of pulses, under the same condition as above. The initial distribution and the excited-state distribution after the recoil cooling are described by $\rho^{\text {th }}$, with $N=25 . \nu T_{\text {sep }}$ is random, taken from a flat distribution that varies in the interval $[0.1,1.1]$.

also checked that by fixing the separation times $T_{\text {sep }}$, the cooling efficiency decreases significatively.

The efficiency of the cooling scheme in populating the ground state depends on the difference between the reexcitation rate of the vibrational ground state and the ones of the first few excited states. To the extent that one can design a confining potential so that the ground state is much more localized in space than the other eigenstates, the cooling efficiency will improve correspondingly. A confining potential satisfying the above requirement could be experimentally realized, for example, by adding to an harmonic trap an additional optical potential. In the following we will assume a potential

$$
V(x)=\frac{1}{2} 2 m \nu^{2}\left[x^{2}+\epsilon \frac{x^{2}}{1+g x^{2}}\right]
$$

where $\epsilon$ and $g$ characterize the shape of the potential near the center. An example of what potential (16) looks like is shown in Fig. 4(a). Figure 4(b) shows the spatial widths of the first 12 eigenstates for $g=2000 / a_{0}^{2}$ and $\epsilon=19 \times 10^{5}$ (curve with $\bigcirc$ ), compared with the corresponding widths of the harmonic-oscillator eigenstates with the same $\nu$ (curve with $\times$ ). For this choice of the parameters $\epsilon$ and $g$, the eigenvectors of the potential tend to the number states of the harmonic oscillator for $n \geqslant 1$. In the present example, the ground state has a spatial width $a_{0} / 5$, and it is thus well localized with respect to the other eigenstates. The laser-cooling scheme works very efficiently in populating the ground state, as may be seen from Fig 5: here the population of the ground state in the trapping potential $V(x)$ is plotted as a function of the number of pulses (solid line) for a doughnut mode with $\alpha=8$ and $2 n=2$ [cf. Eq. (15)]. The Lamb-Dicke parameter for the eigenstates $n \geqslant 1$ and for the internal excited state $|e\rangle$, is $\eta=5$, while the ground state has a spatial width corresponding to an effective Lamb-Dicke parameter $\eta^{\prime}=\eta / 5=1$. We can see that during the cooling almost all atoms are accumulated in the ground state of $V(x)$. The dashed line in Fig. 5 shows, for reference, the evolution of the population of the ground state of a pure harmonic-oscillator potential with $\eta=1$. In this second case, we have used a doughnut 


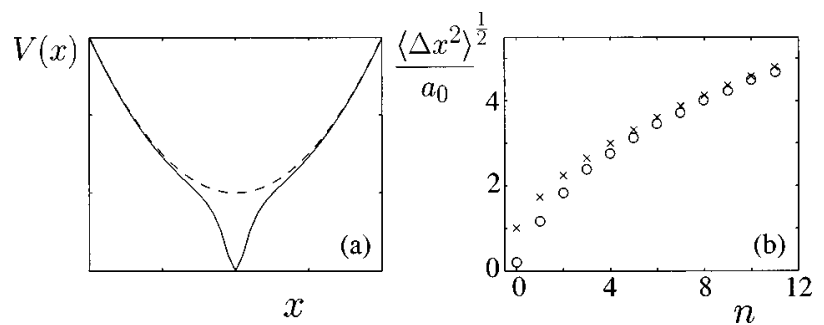

FIG. 4. (a) Plot of $V(x)$ as a function of $x$ for arbitrary values of $\epsilon$ and $g$ (dashed line: harmonic potential). (b) $\left\langle\Delta x^{2}\right\rangle^{1 / 2}$ in units of $a_{0}$ as a function of the first 12 eigenstates of $V(x)$ (curve with $\bigcirc$ ) and of the first 12 ones of the harmonic oscillator potential (curve with $\times$ ), for $\epsilon=19 \times 10^{5}$ and $g=2000 / a_{0}^{2}$. Here $n$ labels the eigenstates by increasing energy.

mode with $2 n=2$ and $\alpha=10$. Note that the sample with $\eta=1$ is colder at the beginning, since its initial distribution is thermalized with $\langle n\rangle \approx 4$.

The result obtained here is rather general, and can be summarized as follows: through the creation of a more localized ground state, obtained by a suitable perturbation of harmonic potential of a given $\eta$, the present cooling mechanism allows us to cool all the atoms to the ground state, even for the Raman pulse intensity distribution in a low order doughnut mode.

\section{CONCLUSIONS}

In this paper we have studied laser cooling of trapped atoms to a single quantum state, based on creating a dark state in position space by reexciting the atoms with a cooling laser which has no spatial overlap with the trap ground state. In contrast to velocity selective coherent population trapping [10], our scheme has been designed for trapped atoms, and therefore it is not limited by the flat-bottom trap condition [16]. In contrast to Raman and sideband cooling, the sidebands do not need to be resolved. The scheme can be applied in the limit of weak confinement $(\Gamma>\nu)$ and outside the Lamb-Dicke regime.

Assuming a doughnut mode as a model of the spatial profile of the laser, our numerical calculations have shown that more than $80 \%$ of the atoms can be cooled to the ground state of a harmonic-oscillator potential. Furthermore, this number can be increased significantly for trapping potentials where the ground state is much better localized than the first few excited vibrational states. The randomization of the separation time in the harmonic-oscillator case is fundamen-

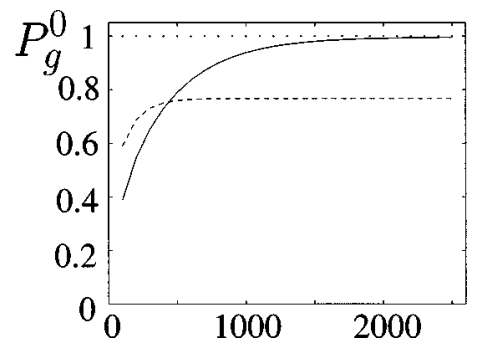

FIG. 5. Ground-state occupation probability vs the number of pulses. Solid line: ground state of atoms trapped by $V(x)$, with $\eta=5$ and $\eta^{\prime}=1, \rho^{\text {th }}$ with $N=25$, and a Raman laser corresponding to a doughnut mode with $2 n=2$ and $\alpha=8$. Dashed line: ground state of a harmonic oscillator with $\eta=1, \rho^{\text {th }}$, with $N=4$, and a Raman laser corresponding to a doughnut mode with $2 n=2$ and $\alpha=10$. In both cases $\max [\Omega(x) \Delta t / 2]=0.6 \pi . \nu T_{\text {sep }}$ is random, taken from a flat distribution that varies in the interval $[0.1,1.1]$.

tal in order to achieve laser cooling to the ground state. The model presented here is one dimensional, but it can be easily extended to two dimensions. In that case, one could use the plane orthogonal to the doughnut mode axis, obtaining similar results as in the one-dimensional case. The reason for that is that the heating processes (due to photon scattering during repumping) we have considered in the one-dimensional case will be similar to the two-dimensional one. Experimentally, the one- and two-dimensional schemes correspond to the employment of a single doughnut mode, whose axis coincides with the one- and two-dimensional trap axes, respectively, and whose dark region is shaped into the size of the one- and two-dimensional trap ground state respectively. The extension to three dimensions could be achieved by crossing two doughnut modes, which propagate in directions that are orthogonal one to the other, and whose axes cross at the center of a three-dimensional trap. In this way a three-dimensional dark region would be created, that could be shaped into the size of the trap ground state. The scheme may be useful for achieving Bose-Einstein condensation with laser cooling, and more in general in preparing with laser cooling a macroscopic occupation of a state of motion.

\section{ACKNOWLEDGMENTS}

G.M. is grateful to J. Eschner for many stimulating discussions. This work was supported by the Austrian Fond zur Förderung der wissenschaftlichen Forschung and the TMR network ERBFMRX-CT96-0002.
[1] A discussion about the advantages of using laser cooling for achieving a macroscopic number of particles in a single quantum state can be found in J. I. Cirac, M. Lewenstein, and P. Zoller, Phys. Rev. Lett. 72, 2977 (1994), and references therein.

[2] A. Kuhn, H. Perrin, W. Hänsel, and C. Salomon, in OSA TOPS on Ultracold Atoms and BEC, edited by K. Burnett (OSA, Washington, DC, 1996), Vol. 7, p. 58.
[3] H. J. Lee, C. S. Adams, M. Kasevich, and S. Chu, Phys. Rev. Lett. 76, 2658 (1996).

[4] M. H. Anderson, J. R. Ensher, M. R. Matthews, C. E. Wieman, and E. A. Cornell, Science 269, 198 (1995); K. B. Davis, M.-O. Mewes, M. R. Andrews, N. J. van Druten, D. S. Durfee, D. M. Kurn, and W. Ketterle, Phys. Rev. Lett. 75, 3969 (1995).

[5] A central obstacle toward achieving the quantum degenerate 
regime with laser cooling is reabsorption of laser photons. See J. I. Cirac, M. Lewenstein, and P. Zoller, Europhys. Lett. 35, 647 (1996); U. Janicke and M. Wilkens, ibid. 35, 561 (1996), and references cited therein.

[6] F. Diedrich, J. C. Berquist, W. M. Itano, and D. J. Wineland, Phys. Rev. Lett. 62, 403 (1989); C. Monroe, D. M. Meekhof, B. E. King, S. R. Jefferts, W. M. Itano, D. J. Wineland, and P. Gould, ibid. 75, 4011 (1995).

[7] S. Stenholm, Rev. Mod. Phys. 58, 699 (1986); R. Blatt, in Fundamental Systems in Quantum Optics, Proceedings of the Les Houches Summer School, Session LIII, edited by J. Dalibard, J. M. Raimond, and J. Zinn-Justin (Elsevier, Amsterdam, 1992).

[8] J. I. Cirac, A. S. Parkins, R. Blatt, and P. Zoller, Adv. At., Mol., Opt. Phys. 37, 237 (1996).

[9] G. Morigi, J. I. Cirac, M. Lewenstein, and P. Zoller, Europhys. Lett. 39, 13 (1997).

[10] A. Aspect, E. Arimondo, R. Kaiser, N. Vansteenkiste, and C. Cohen-Tannoudji, Phys. Rev. Lett. 61, 826 (1988); J. Lawall, S. Kulin, B. Saubamea, N. Bigelow, M. Leduc, and C. CohenTannoudji, ibid. 75, 4194 (1995).

[11] M. Kasevich and S. Chu, Phys. Rev. Lett. 69, 1741 (1992); N. Davidson, H. Lee, M. Kasevich, and S. Chu, ibid. 72, 3158 (1994); J. Reichel, O. Morice, G. M. Tino, and C. Salomon,
Europhys. Lett. 28, 477 (1994).

[12] The idea of a spatial region where no photons are present is also an essential element in the "dark SPOT" [W. Ketterle, K. B. Davis, M. A. Joffe, A. Martin, and D. E. Pritchard, Phys. Rev. Lett. 70, 2253 (1993)], and sideband cooling of ions with standing light waves [J. I. Cirac, R. Blatt, P. Zoller, and W. D. Phillips, Phys. Rev. A 46, 2668 (1992)].

[13] L. Allen, M. W. Beijersbergen, R. J. C Spreeuw, and J. P. Woerdman, Phys. Rev. A 45, 8185 (1992); M. W. Beijersbergen, L. Allen, H. E. L. O. van der Veen, and J. P. Woerdman, Opt. Commun. 96, 123 (1993); S. Kuppens, M. Rauner, M. Schiffer, G. Wokurka, T. Slawinski, M. Zinner, K. Sengstock, and W. Ertmer, in OSA Trends in Optics and Photonics (TOPS), edited by Keith Burnett (OSA, Washington, DC, 1996), Vol. 7; M. Schiffer, M. Rauner, S. Kuppens, M. Zinner, K. Sengstock, and W. Ertmer (unpublished).

[14] J. Dalibard, in Proceedings of ZICAP, edited by H. B. van Linden van den Heuvell, J. T. M. Walraven, and M. W. Reynolds (World Scientific, Singapore, 1997).

[15] C. W. Gardiner, Quantum Noise (Springer-Verlag Berlin, 1991).

[16] R. Dum, P. Marte, T. Pellizzari, and P. Zoller, Phys. Rev. Lett. 73, 2829 (1994); T. Pellizzari, P. Marte, and P. Zoller, Phys. Rev. A 52, 4709 (1995). 